\title{
Gestión ambiental y su influencia en la fiscalización ambiental de la Municipalidad Provincial de San Martín
}

Environmental management and its influence on the environmental control of the Provincial Municipality of San Martín

Gestão ambiental e sua influência no controle ambiental do Município Provincial de San Martín

Mirko Juniors Morales Ramírez

mijuproducciones@gmail.com

Instituto de Gobierno y de Gestión Pública de la Universidad San Martín de Porres - Perú

ORCID iD N0000-0002-8593-2346

Armando Figueroa Sánchez

armanfig.iggp@gmail.com

Instituto de Gobierno y de Gestión Pública de la Universidad San Martín de Porres - Perú

ORCID iD N0000-0003-1891-9739

\begin{abstract}
RESUMEN
El objetivo de la investigación fue analizar de qué forma, la gestión ambiental influye en la fiscalización ambiental de la Municipalidad Provincial de San Martin. El enfoque fue cuantitativo, no experimental, descriptivo, de nivel correlacional, con una muestra de 76 servidores de la Municipalidad. Entre los resultados se encontró que, para el 92,1\% de los servidores entrevistados, en la Municipalidad no se facilita el acceso a la justicia ambiental. Asimismo, para el 89,5\% de los servidores, la municipalidad no siempre evalúa el desempeño de la regulación ambiental. Para el 97,4\% de los servidores, la municipalidad no siempre, genera la información ambiental para la toma de decisiones. Para el $92,1 \%$, la municipalidad no siempre toma las medidas para garantizar un ambiente sano (agua, aire, suelo, residuos sólidos). Por su parte, el 97,4\% indicó que, la municipalidad no siempre promociona adecuadamente la inversión sostenible. El 92,1\% indicó que, la municipalidad no siempre gestiona su territorio aprovechando los recursos naturales. Asimismo, el 94,7\%, sostiene que las evidencias técnicas en la fiscalización ambiental no son eficientes. Por su parte, el $100 \%$ de los entrevistados, señaló que, el cumplimiento de las obligaciones ambientales en el proceso de fiscalización no es eficiente. Para el 81,6\%, el estudio de las posibles infracciones administrativas a nivel ambiental no es eficiente. Según el 97,4\%, la implementación de las medidas correctivas según la fiscalización ambiental no es eficiente. Se concluye que, la gestión ambiental se encuentra asociada a la fiscalización ambiental de la Municipalidad Provincial de San Martin.
\end{abstract}

Palabras clave: Gestión, Ambiental, Fiscalización.

\begin{abstract}
The objective of the research was to analyze how environmental management influences the environmental control of the Provincial Municipality of San Martin. The approach was quantitative, non-experimental, descriptive, correlational level, with a sample of 76 employees of the Municipality. Among the results, it was found that, for $92.1 \%$ of the servers interviewed, the Municipality does not provide access to environmental justice. Likewise, for $89.5 \%$ of the employees, the municipality does not always evaluate the performance of environmental regulation. For $97.4 \%$ of the servers, the municipality does not always generate environmental information for decision making. For $92.1 \%$, the municipality does not always take measures to guarantee a healthy environment (water, air, soil, solid waste). For their part, $97.4 \%$ indicated that the municipality does not always adequately promote sustainable investment. $92.1 \%$ indicated that the municipality does not always manage its territory taking advantage of natural resources. Likewise, $94.7 \%$ maintain that the technical evidence in environmental control is not efficient. On the other hand, $100 \%$ of those interviewed indicated that compliance with environmental obligations in the inspection process is not efficient. For $81.6 \%$, the study of possible administrative infractions at the environmental level is not efficient. According to $97.4 \%$, the implementation of corrective measures according to environmental control is not efficient. It is concluded that environmental management is associated with the environmental control of the Provincial Municipality of San Martin.
\end{abstract}

Keywords: Management, Environmental, Control.

\section{RESUMO}

O objetivo da pesquisa foi analisar como a gestão ambiental influencia o controle ambiental do Município Provincial de San Martin. A abordagem foi quantitativa, não experimental, descritiva, de nível correlacional, com uma amostra de 76 
funcionários do Município. Entre os resultados, constatou-se que, para 92,1\% dos servidores entrevistados, o Município não oferece acesso à justiça ambiental. Da mesma forma, para 89,5\% dos funcionários, o município nem sempre avalia o desempenho da regulação ambiental. Para 97,4\% dos servidores, nem sempre o município gera informações ambientais para tomada de decisão. Para 92,1\%, o município nem sempre toma medidas para garantir um ambiente saudável (água, ar, solo, resíduos sólidos). Por sua vez, 97,4\% indicaram que o município nem sempre promove adequadamente o investimento sustentável. 92,1\% indicaram que o município nem sempre administra seu território aproveitando os recursos naturais. Da mesma forma, 94,7\% sustentam que a comprovação técnica no controle ambiental não é eficiente. Por outro lado, $100 \%$ dos entrevistados indicaram que o cumprimento das obrigações ambientais no processo de fiscalização não é eficiente. Para 81,6\%, o estudo de possíveis infrações administrativas em nível ambiental não é eficiente. Segundo $97,4 \%$, a implementação de medidas corretivas de acordo com o controle ambiental não é eficiente. Conclui-se que a gestão ambiental está associada ao controle ambiental do Município Provincial de San Martin.

Palavras-chave: Gestão, Meio Ambiente, Fiscalização.

\section{INTRODUCCIÓN}

Con el paso de los años es cada vez más evidente la dependencia del ser humano al medio ambiente, y las vulnerabilidades ante los grandes cambios climáticos, y el deterioro del medio ambiente; por ello, a nivel global se han desarrollado estrategias y planes para la conservación. Sin embargo, en Latinoamérica según (Gutiérrez, 2020) ha costado un poco más unificar los aspectos del medio ambiente con la salud de la población, aunque existen "políticas, programas y estrategias de salud ambiental, estos cuentan con disímiles niveles de jerarquía y aplicabilidad, y en términos generales hay que desarrollar un proceso deductivo para llegar a establecer la relación entre factores del medioambiente y de la salud". En Perú, a fin de garantizar la calidad de vida de las personas y al desarrollo sostenible del país, el Estado promulgó la (Resolución Ministerial N²47-2013-MINAM, 2013), para regular el Régimen de Fiscalización Ambiental estableciendo lineamientos, principios y bases comunes de la fiscalización ambiental en el país, así como las disposiciones generales que deben cumplir de manera obligatoria las Entidades de Fiscalización Ambiental (EFA). Dicha norma es concordante con la (Ley General del Ambiente, 2017), que regula que toda persona, que genere impactos ambientales significativos, en otras palabras, todas las figuras que tengan alguna influencia en el ambiente están sometida a las acciones de fiscalización y control ambiental. En tal sentido, el (Organismo de Evaluación y Fiscalización Ambiental, 2012) sostiene que las Entidades de Fiscalización Ambiental Nacional (EFA) "son aquellas con facultades expresas para desarrollar funciones de fiscalización ambiental". Durante el periodo de confinamiento a causa de la pandemia de COVID-19 las condiciones ambientales mejoraron en ciertos aspectos, sobre todo en la calidad del aire, en tal sentido (Oyague, Yaja, \& Franco, 2020) sostienen que, "la reducción de actividad humana, por el aislamiento, produce una serie de impactos positivos sobre el ambiente, pero estos son de corta duración”, para (Oyague, Yaja, \& Franco, 2020) uno de los riesgos naturales posterior al estado de confinamiento, es el acelerado deterioro del medio ambiente, por la necesidad de recuperar las pérdidas económicas.

Entre las problemáticas ambientales se advierte el incumplimiento a las medidas de protección, esto se puede inferir por el informe del (Instituto Nacional de Estadística e Informática, 2020) en el que se precisa que, "durante el año 2019, se registraron 447 delitos en contra del medio ambiente; por contaminación (250) e incumplimiento de normas sanitarias (58), entre los más frecuentes". Uno de los recursos más afectados es la calidad del aire, conforme al (Instituto Nacional de Estadística e Informática, 2020) "fueron registrados 2 millones 316 mil atenciones a niños menores de 5 años afectados por infecciones respiratorias". Por otro lado, entre las regiones más afectadas por las fuertes precipitaciones se encuentra "Loreto (2 mil 965 milímetros), Ucayali (2 mil 696 milímetros), Madre de Dios (2 mil 8 milímetros), San Martín (1 mil 722 milímetros) y Pasco (1 mil 633 milímetros)" (Instituto Nacional de Estadística e Informática, 2020). Dentro de este orden de ideas, el (Programa de Política y Gestión Ambiental - SPDA, 2016) plantea que, la evaluación de impacto ambiental es una herramienta que busca reducir la afectación al ambiente, a través de un método administrativo para reducir los impactos provocados por ocupaciones humanas. 
En tal sentido, es necesario precisar que la EFA responsable de realizar acciones de fiscalización ambiental por problemáticas ambientales (contaminación sonora, residuos sólidos) en el distrito de Tarapoto, es la Municipalidad Provincial de San Martin, por lo que es necesario conocer como la gestión influye en las acciones de fiscalización ambiental, para ello se plantearon los siguientes problemas y objetivos científicos:

Problema General: PG.- ¿De qué forma, la gestión ambiental influye en la fiscalización ambiental de la Municipalidad Provincial de San Martin, 2021?

Problemas Específicos: PE1.- ¿Cómo la gestión ambiental influye en la evaluación de la Municipalidad Provincial de San Martin, 2021?, PE2.- ¿De qué manera, la gestión ambiental influye en la supervisión de la Municipalidad Provincial de San Martin, 2021?, PE3.- ¿Cómo la gestión ambiental influye en la potestad sancionadora de la Municipalidad Provincial de San Martin, 2021?

Objetivo General: OG. - Analizar de qué forma, la gestión ambiental influye en la fiscalización ambiental de la Municipalidad Provincial de San Martin, 2021.

Objetivos Específicos: OE1.- Conocer cómo la gestión ambiental influye en la evaluación de la Municipalidad Provincial de San Martin, 2021; OE2.- Establecer de qué manera, la gestión ambiental influye en la supervisión de la Municipalidad Provincial de San Martin, 2021; OE3.Identificar cómo la gestión ambiental influye en la potestad sancionadora de la Municipalidad Provincial de San Martin, 2021.

Las implicancias de conocer la gestión y fiscalización ambiental de la Municipalidad Provincial de San Martin, permitirá analizar el cumplimiento del Régimen de Fiscalización Ambiental, cuyos resultados buscan mejorar la reducción de los problemas de la comunidad asociados a los residuos sólidos. En este mismo sentido, (Armas, 2020) sostiene que, "las municipalidades deben jugar un rol muy importante como ente impulsor de políticas ambientales a nivel local, procurando que la gestión ambiental del municipio tenga la capacidad de enfrentarse a grandes desafíos en la búsqueda de un verdadero desarrollo sostenible".

Asimismo, la investigación sirve como marco de referencia y de apertura para futuras investigaciones dentro y fuera de la municipalidad Provincial de San Martin. En otras palabras, se estuvo contribuyendo en la ejecución de las actividades de fiscalización ambiental para disminuir la contaminación sonora y de residuos sólidos en la jurisdicción territorial de la ciudad de Tarapoto. Siendo viable porque se contó con acceso a la información de la gestión ambiental de la Municipalidad Provincial de San Martin. Asimismo, se contó con los recursos financieros y de tiempos, para llevar a cabo el desarrollo de los objetivos de la investigación.

\section{FUNDAMENTO TEÓRICO}

\subsection{Investigaciones previas}

En Colombia, (Roggeroni, 2014) en el "Análisis de la Aplicación del Sistema Local de Gestión Ambiental de la Provincia de Mariscal Ramón Castilla partir de la Norma ISO 14001", cuyo objetivo fue analizar los elementos de la implementación de la gestión ambiental, mediante una metodología cuantitativa de carácter descriptivo. (Roggeroni, 2014) concluyó que, "la Municipalidad aplica el Sistema de Gestión Ambiental a partir de la Norma Internacional Estandarizada ISO 14001 y cuenta con un 51\% de instrumentos de gestión ambiental, pero aun así no pudo resolver las falencias cualitativas de planificación y que son susceptibles de mejorar".

En la investigación de (Ramírez, 2017) titulada "Importancia de la fiscalización ambiental en los factores que originan la aplicación de la sanción en materia ambiental firme en los procedimientos sancionadores de la Municipalidad Provincial de Tambopata Departamento de Madre de Dios", desarrolló como objetivo conocer la importancia de la fiscalización ambiental en la 
municipal, dicha investigación, fue de un enfoque cualitativo, de nivel descriptivo. En materia de supervisión $71 \%$ fueron por manejo de residuos sólidos y $29 \%$ acciones de supervisión por contaminación ambiental. Las ordenanzas que establecen las, carecen de especificación para detallar las funciones en materia de fiscalización ambiental; así mismo (Ramírez, 2017) sostiene que, existen procedimientos administrativos sancionadores que se inician en la Municipalidad, pero carecen de criterios técnicos y legales que respalden las resoluciones de sanción, estas terminan cayendo en nulidad por distintos vicios legales.

Además (Gómez, 2017) en su tesis "La fiscalización ambiental del OEFA: características de un modelo de ejercicio de la potestad sancionadora que armoniza con la inversión". Cuyo objeto de la investigación fue demostrar que durante el periodo comprendido entre finales del año 2012 e inicios del año 2016, el ejercicio de la potestad sancionadora del Organismo de Evaluación y Fiscalización Ambiental fue razonable, proporcional y cumplió los estándares más altos exigidos para la adecuada protección del derecho de defensa y el debido procedimiento, concluyó que es posible armonizar la potestad sancionadora en materia ambiental con el desarrollo de las actividades económicas, especialmente aquellas vinculadas a las actividades extractivas de recursos naturales (minería, hidrocarburos, pesquería, entre otras).

Por otro lado, (Araujo \& Casanova, 2018) en la investigación sobre "El grado de cumplimiento de la fiscalización ambiental en las municipalidades distritales de la provincia de Trujillo durante los años 2013 al 2017”, cuyo objetivo fue establecer el grado de cumplimiento de la fiscalización ambiental en las municipalidades, la investigación, tuvo un enfoque mixto. (Araujo \& Casanova, 2018) concluyó que, "el 29\% cuenta con el presupuesto económico para el desarrollo de funciones de fiscalización ambiental, mientras que, el 71\% no tiene dicha asignación.

Es así que (González, 2018) en su estudio "Evaluación integral del proceso de fiscalización ambiental realizado por el Organismo de Evaluación Ambiental en el Perú", se desarrolla con la finalidad de evaluar el proceso de fiscalización del Organismo de Evaluación y Fiscalización Ambiental (OEFA), en base a la necesidad de fortalecer la gestión ambiental para lograr una fiscalización ambiental efectiva, concluyendo que se identificaron los indicadores de gestión del OEFA, en cuanto a acciones de fiscalización ambiental. El indicador se basa en el número de resoluciones, dándose aproximadamente más de mil doscientos sesenta (1260) resoluciones en lo que va del año 2017. Dentro de los factores que favorecieron al cumplimiento de la meta física, se ha resaltado el compromiso de los colaboradores del OEFA, la organización en el trabajo, así como la experiencia con la que cuentan en el campo de sus competencias.

En la tesis de (Roca, 2019) titulada "Auditoría ambiental y su efecto en la evaluación de la gestión ambiental de la Municipalidad Provincial de Huamanga, 2018”, se cumplió con el objeto de "determinar que la auditoría ambiental y su efecto en la evaluación de la gestión ambiental", a través del enfoque cuantitativo, correlacional. En los resultados se pudo observar que, el $73 \%$ afirman que la toma de decisiones si se tiene en cuenta la repercusión de sus actividades ambientales en la sociedad y el $27 \%$ respondieron que no se tiene en cuenta la repercusión de sus actividades ambientales en la sociedad. Por otro lado, el $41 \%$ afirman que siempre reciben capacitación en materia ambiental; el 39\% respondieron que ocasionalmente; el 10\% respondieron que a veces, y el $10 \%$ respondieron que nunca reciben capacitación en materia ambiental. (Roca, 2019) concluyó que, la auditoría ambiental influye en el perfeccionamiento de buenas prácticas en la Municipalidad.

En la investigación de (Ushiñahua, 2019) sobre la "Gestión ambiental y su relación con el manejo integral de residuos sólidos en la Municipalidad Provincial de San Martín, 2018”, cuyo objetivo principal fue establecer la asociación entre los elementos de estudio, utilizando la metodología descriptiva, correlacional. (Ushiñahua, 2019) presenta en los resultados del estudio que, el " $64 \%$ de los resultados calificó a la dimensión fiscalización como Inadecuada". Concluyendo que, "existe relación directa entre la gestión ambiental y manejo de los residuos 
sólidos en la Municipalidad Provincial de San Martin; con un coeficiente de correlación de 0,689" (Ushiñahua, 2019).

Además, (Tovalino 2019) en su tesis "Gestión ambiental y conciencia ambiental de los trabajadores de la Dirección Regional de Agricultura Junín, 2019”, señala que la investigación tuvo como objetivo general, determinarla la relación entre la gestión ambiental y conciencia ambiental de los trabajadores de la Dirección Regional de Agricultura Junín, 2019. La población es de 114 administrativos, es no probabilístico, en los cuales se han empleado la variable: Gestión ambiental y la conciencia ambiental. Es una investigación de tipo básica desarrollada en el enfoque metodológico cuantitativo de método científico no experimental, de nivel descriptiva correlacional, el enfoque cuantitativo porque sostenida es en escala ordinal, obteniendo un alto grado de confiabilidad y validez de los instrumentos de recopilación de datos, realizados con el soporte estadístico del programa SPSS versión 25.0 y la opinión o juicio de expertos. Concluye que el valor de significancia asociada a la prueba es de 0.000 inferior al valor de significancia de la prueba, por lo que podemos rechazar la hipótesis nula y afirmamos que las variables gestión ambiental y la conciencia ambiental presentan una relación directa y el coeficiente de correlación de Rho de Spearman alcanza el 0.746 , podemos afirmar que la relación es directa y alta.

Por su parte, (Rodríguez, 2020) realizó una investigación denominada "Gestión ambiental y ecoeficiencia en la Municipalidad Provincial de Moyobamba, 2020", cuyo objetivo fue determinar la asociación entre las variables utilizando el método correlacional. Entre los resultados se pudo apreciar que, el 61,6\% consideró que la gestión ambiental en la municipalidad es regular, el 20,5\% que es malo y el 17,8\% buena. En cuanto al servicio social el 46,6 opinó que es regular y el 31,5\% que es malo. (Rodríguez, 2020) concluyó que, entre las variables existe "correlación positiva moderada y significativa. El valor del coeficiente de correlación Rho Spearman fue de 0.595".

En la investigación de (Rosado, 2021) sobre la "Influencia de la fiscalización ambiental en la gestión sostenible de los recursos naturales del Gobierno Regional de Ucayali 2018", cuyo objetivo principal fue determinar en qué medida la fiscalización ambiental influirá en la gestión sostenible de los recursos naturales del Gobierno Regional de Ucayali, la fiscalización ambiental a pesar de estar normado para su aplicación por parte del Gobierno Regional de Ucayali no tiene los efectos necesarios, se denota la falencia en cuanto al análisis de las funciones de evaluación, supervisión y fiscalización lo cual es un problema en la organización de la administración práctica dentro del territorio con competencia ambiental y de recursos naturales. La investigación en cuanto a su metodología es cuantitativa de nivel correlacional, de acuerdo a los resultados de la investigación la fiscalización ambiental y la gestión sostenible de los recursos naturales, (Rosado, 2021) concluye que la fiscalización ambiental tiene relación significativa en la gestión sostenible de los recursos naturales del Gobierno Regional de Ucayali.

\subsection{Bases teóricas}

\subsubsection{Gestión ambiental}

La gestión ambiental conforme a la (Ley General del Ambiente, 2017) se define como "un proceso continuo, constituido por el conjunto estructurado de principios, normas técnicas, procesos y actividades, orientado a administrar los intereses, expectativas y recursos relacionados con los objetivos de la política ambiental y alcanzar así, una mejor calidad de vida".

La (Comisión Multisectorial, 2012) presentó un grupo de acciones orientadas a mejorar el enfoque ambiental y social del desarrollo en el territorio, estas se centran en cuatro ejes estratégicos "estado soberano y garante de derechos (gobernanza / gobernabilidad), Mejora en la calidad de vida con ambiente sano, Compatibilizando el aprovechamiento armonioso de los recursos naturales, Patrimonio natural saludable". Estos ejes manifiestan la prioridad nacional desde la perspectiva ambiental, incorporando correctamente los retos para garantizar la calidad ambiental. 
Paralelamente, dichos ejes organizan la mediación estatal en la administración ambiental, explicando fines claros; y asegurando el cumplimiento de las políticas y normativas. Según la (Comisión Multisectorial, 2012) el primer eje estratégico es la gobernabilidad, el cual se entiende como la voluntad política para garantizar el cumplimiento de sus funciones en torno al acceso a la justicia y fiscalización ambiental, mejorar el desempeño de la gestión y regulación ambiental. Para el desarrollo de este eje se consideran las siguientes prioridades: "acceso a la justicia y a la fiscalización ambiental, mejorar el desempeño del estado en la gestión y regulación ambiental, generar información ambiental sistémica e integrada para la toma de decisiones, fortalecer la educación ambiental, construcción de capacidades y profesionalización ambientales" (Comisión Multisectorial, 2012).

El segundo eje consiste en la calidad de vida con ambiente sano, en este sentido, la (Comisión Multisectorial, 2012) sostiene que, "el Estado debe velar por la prevención y mitigación de las externalidades negativas que generan las actividades económicas, protegiendo la salud de las personas y de los ecosistemas", con la finalidad de garantizar un ambiente sano. El tercer eje trata del aprovechamiento de los recursos naturales, "se constituye en un impulso que se asocia con la gestión sostenible de los recursos naturales como elemento fundamental de las políticas y estrategias de desarrollo nacional". Para este eje se considera la promoción de inversiones sostenibles, la gestión del territorio, actividades productivas y desarrollo con inclusión social. (Comisión Multisectorial, 2012). El cuarto eje es denominado patrimonio natural saludable, este eje es entendido como el mantenimiento y mejoramiento de la función de los ecosistemas para proporcionar bienes y servicios del medio ambiente, como regulación del clima, regulación del periodo del agua, productos forestales, pesqueros, suelo agrícola, entre otros, de manera aseguren las condiciones idóneas para conseguir la paz humano, el aumento y el desarrollo, en beneficio poblacional (Comisión Multisectorial, 2012).

\subsubsection{Fiscalización ambiental}

La fiscalización ambiental según el (Organismo de Evaluación y Fiscalización Ambiental, 2018) "es la acción de control que realiza una entidad pública para verificar el cumplimiento de las obligaciones ambientales", comprende la evaluación, supervisión, y potestad Sancionadora. En otras palabras, la fiscalización ambiental tiene como objetivo garantizar que los administrados cumplan con sus obligaciones, a efectos de asegurar la conservación del ambiente y el aprovechamiento sustentable de los recursos naturales.

El (Organismo de Evaluación y Fiscalización Ambiental, 2018) sostiene la evaluación radica en la elaboración de acciones de monitoreo de la calidad del ambiente, y el estado de conservación de los recursos naturales. El objetivo principal es la obtención de pruebas técnicas del estado de los elementos del medio ambiente. También, puede evaluar los diversos factores determinantes de la calidad del ambiente, tales como los derramamientos, el deterioro del medio ambiente, entre otros.

La función de supervisión según el (Organismo de Evaluación y Fiscalización Ambiental, 2018) consiste en las "acciones de verificación y seguimiento con el propósito de asegurar el cumplimiento de las obligaciones ambientales que tienen a su cargo los administrados; es decir, a través de esta función, se verifica el cumplimiento de las obligaciones ambientales fiscalizables".

Finalmente, entre las funciones de la fiscalización ambiental se encuentra la potestad sancionadora, la cual según el (Organismo de Evaluación y Fiscalización Ambiental, 2018) alcanza la "facultad de investigar las posibles infracciones administrativas e imponer sanciones y medidas administrativas por el incumplimiento de obligaciones ambientales fiscalizables en el marco de un procedimiento administrativo sancionador". Por su lado, el (Organismo de Evaluación y Fiscalización Ambiental, 2018) señala que, las medidas correctivas reducen el impacto perjudicial de las infracciones en el cuidado del medio ambiente. El método administrativo sancionador debe respetar las garantías y derechos de los administrados. 


\section{PROCEDIMIENTOS METODOLOGICOS}

El presente estudio tuvo un enfoque cuantitativo, no experimental, descriptivo, de nivel correlacional, dado que es un estudio que tiene como propósito evaluar la relación que exista entre dos o más conceptos, categorías o variables (Hernández, et al (2003) p. 121); se recolectó la información de manera directa, y confiable en la realidad donde se presenta, es decir la fuente fueron los servidores de la Municipalidad Provincial de San Martín, de esta manera se observó el fenómeno en su contexto objetivo, sin influenciar o manipular variables. La población estuvo conformada por 233 servidores de la Municipalidad Provincial de San Martín, según el cuadro de asignación de personal de la (Municipalidad Provincial de San Martín, 2020), sin embargo, una vez realizado el muestreo probabilístico, se estimó que la muestra estuvo representada por 76 servidores de la Municipalidad Provincial de San Martín.

Para efectos de la presente investigación la técnica utilizada fue la encuesta, mediante un cuestionario como instrumento dirigido a los servidores de la Municipalidad Provincial de San Martín. Este cuestionario de tipo survey, fue un documento formado por un conjunto de preguntas redactadas de forma coherente, y organizadas, secuenciadas y estructuradas, con el fin de que sus respuestas ofrezcan toda la información necesaria (Hernández Sampieri, 1997); este cuestionario estuvo conformado por diecinueve (19) interrogantes, una por cada indicador propuesto en la matriz de operacionalización, las respuestas se evaluaron a través de la escala de Likert. La primera parte del cuestionario buscó analizar la variable gestión ambiental con la escala de (nunca, casi nunca, a veces, casi siempre, siempre) y la segunda parte del cuestionario contiene interrogantes sobre la fiscalización ambiental, y se evaluó con la escala de (muy deficiente, deficiente, regular, eficiente, muy eficiente).

La validación del instrumento fue a través de la técnica del juicio de 3 expertos. En cuanto a la confiabilidad del instrumento, fue realizada a través del Alfa de Cronbach, el mismo que según, (George \& Mallery, 2020), es una regla general que se aplica a la mayoría de las situaciones y que una vez aplicado se determinó un valor de 0,941 lo que indica que, el instrumento es excelente. Respecto al aspecto ético del cuestionario, se contó con el consentimiento previo, libre e informado de los 76 servidores de la Municipalidad Provincial de San Martín y además en la investigación no hubo conflicto de interés por parte del autor y asesor con la entidad involucrada, también se respetaron los principios éticos y criterios establecidos en el "Manual para la elaboración de las tesis y los trabajos de investigación para obtener los Grados Académicos de Maestro y Doctor del Instituto de Gobierno y de Gestión Pública de la Universidad San Martín de Porres - Perú"; así mismo se respetó la autoría de las fuentes de información consultadas, empleando las normas APA vigentes, cumpliendo con el rigor científico de la ciencia.

El procesamiento de la información, se realizó utilizando las técnicas de gestión y estadísticas del Software estadístico SPSS versión 26. Para ello, se creó una base de datos, que permitió procesar la información obtenida mediante de la aplicación de los cuestionarios a los servidores de la Municipalidad Provincial de San Martín. Posteriormente se analizaron los resultados de forma descriptiva, y se determinó la influencia de las variables entre sí, mediante las técnicas de correlación del Software estadístico SPSS versión 26.

\section{RESULTADOS Y DISCUSIÓN}

\subsection{Resultados sobre la gestión ambiental de la Municipalidad Provincial de San Martin}

A continuación, se presentan los resultados correspondientes a la gestión ambiental y sus dimensiones (Gobernabilidad, calidad de vida con ambiente sano, aprovechamiento de los recursos naturales y patrimonio natural saludable), según los servidores de la Municipalidad Provincial de San Martín. 
Se encontró en la Tabla 1 y Figura 1 que, para el 5,3\% y el 36,8\% de los servidores de la Municipalidad Provincial de San Martín, la municipalidad nunca y casi nunca realiza una adecuada gestión ambiental. Mientras que, el 57,9\% expresó que, a veces realizan una adecuada gestión ambiental.

\section{Tabla 1}

Gestión ambiental

\begin{tabular}{cccc}
\hline Valor cualitativo & Frecuencia & Porcentaje & Porcentaje acumulado \\
\hline Nunca & 4 & 5,3 & 5,3 \\
Casi nunca & 28 & 36,8 & 42,1 \\
A veces & 44 & 57,9 & 100,0 \\
\hline Total & $\mathbf{7 6}$ & $\mathbf{1 0 0 , 0}$ & \\
\hline
\end{tabular}

\section{Figura 1}

Gestión ambiental

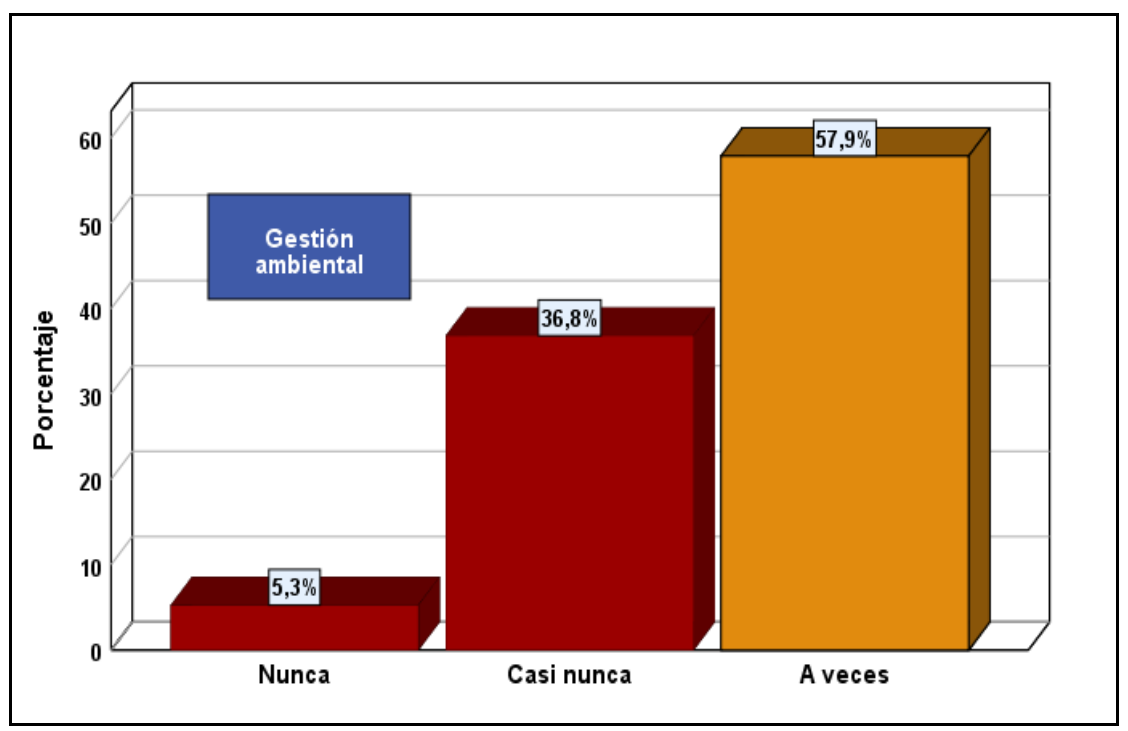

Se observa en la Tabla 2 y Figura 2 el promedio general de la gestión ambiental y sus dimensiones, encontrándose un promedio general 2,4 (Casi nunca), indicando la poca gestión ambiental por parte de la Municipalidad Provincial de San Martin.

\section{Tabla 2}

Promedio general de la gestión ambiental y sus dimensiones

\section{Figura 2}

\begin{tabular}{llc}
\hline Gobernabilidad & 2,5 & A veces \\
Calidad de vida con ambiente sano & 2,4 & Casi nunca \\
Aprovechamiento de los recursos naturales & 2,3 & Casi nunca \\
Patrimonio natural saludable & 2,4 & Casi nunca \\
\hline Gestión ambiental & $\mathbf{2 , 4}$ & Casi nunca \\
\hline
\end{tabular}

Promedio general de la gestión ambiental y sus dimensiones 


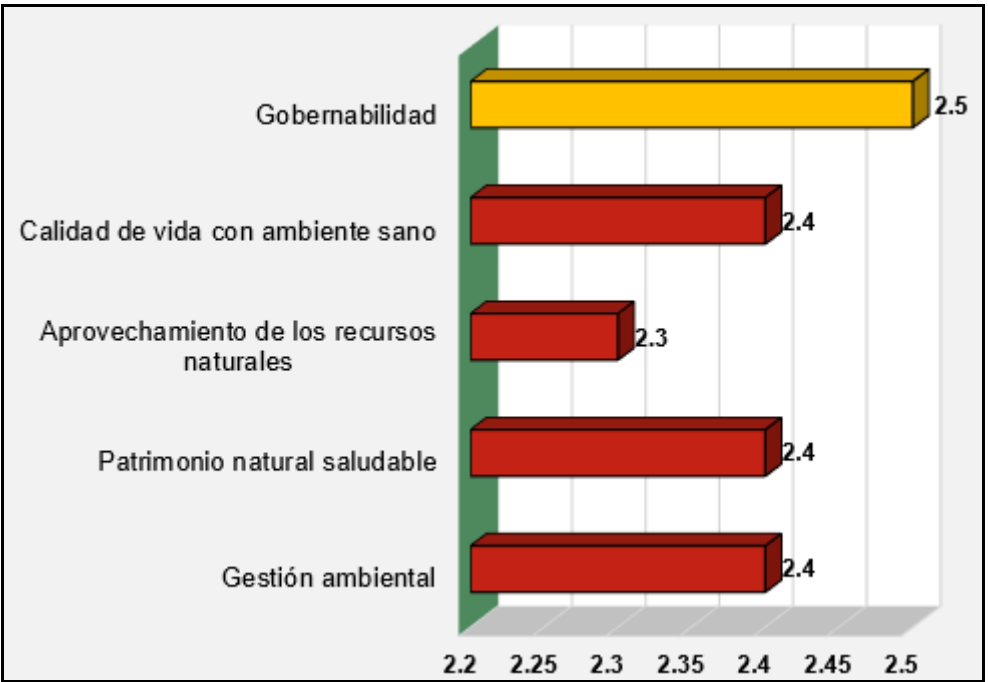

\subsection{Resultados sobre la fiscalización ambiental de la Municipalidad Provincial de San Martin}

A continuación, se presentan los resultados correspondientes a la fiscalización ambiental y sus dimensiones (evaluación, supervisión y potestad sancionadora), según los servidores de la Municipalidad Provincial de San Martín. Se encontró en la Tabla 3 y Figura 3 que, para el 5,3\% y el $50 \%$ de los servidores de la Municipalidad Provincial de San Martín, la fiscalización ambiental se realiza de manera muy deficiente y deficiente respectivamente. El 39,4\% expresó que, se realiza de manera regular, mientras que, solo para el 5,3\% de los servidores, la municipalidad realiza la fiscalización ambiental de manera eficiente.

\section{Tabla 2}

Fiscalización ambiental

\begin{tabular}{lccc}
\hline \multicolumn{1}{c}{ Valor cualitativo } & Frecuencia & Porcentaje & Porcentaje acumulado \\
\hline Muy deficiente & 4 & 5,3 & 5,3 \\
Deficiente & 38 & 50,0 & 55,3 \\
Regular & 30 & 39,4 & 94,7 \\
Eficiente & 4 & 5,3 & 100,0 \\
\hline Total & $\mathbf{7 6}$ & $\mathbf{1 0 0 , 0}$ & \\
\hline
\end{tabular}

\section{Figura 3}

\section{Fiscalización ambiental}

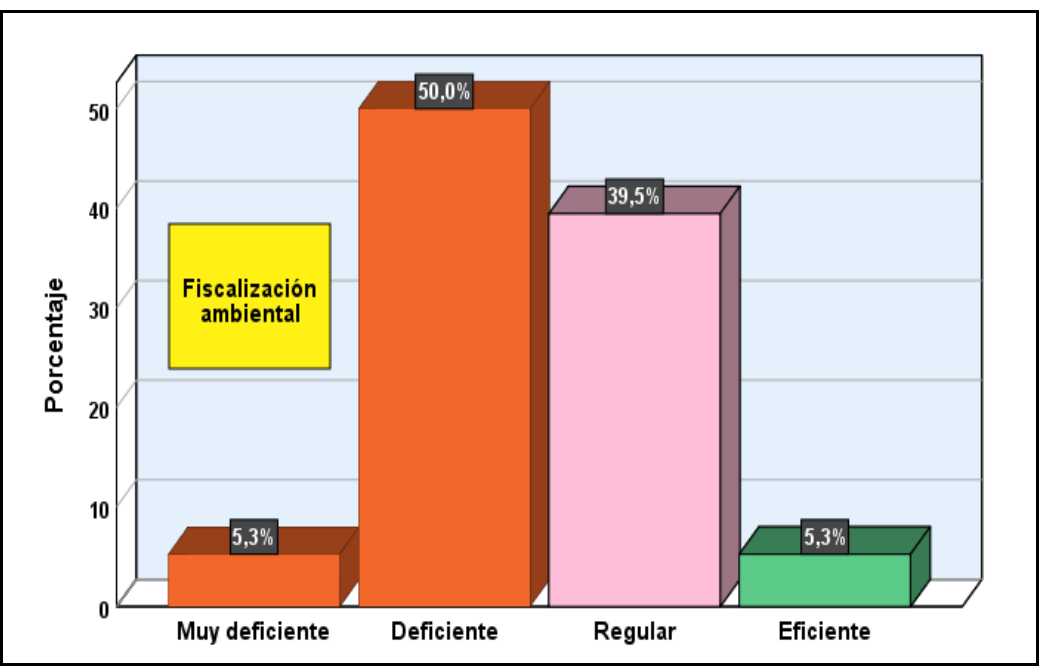


Se muestra en la Tabla 4 y Figura 4 el promedio general de la fiscalización ambiental y sus dimensiones, encontrándose un promedio general 2,4 (Deficiente), indicando la deficiente fiscalización por parte de la Municipalidad Provincial de San Martin.

\section{Tabla 4}

Promedio general de la fiscalización ambiental y sus dimensiones

\begin{tabular}{lll}
\hline Evaluación & 2,4 & Deficiente \\
Supervisión & 2,4 & Deficiente \\
Potestad Sancionadora & 2,3 & Deficiente \\
\hline Fiscalización ambiental & $\mathbf{2 , 4}$ & Deficiente \\
\hline
\end{tabular}

\section{Figura 4}

Promedio general de la fiscalización ambiental y sus dimensiones

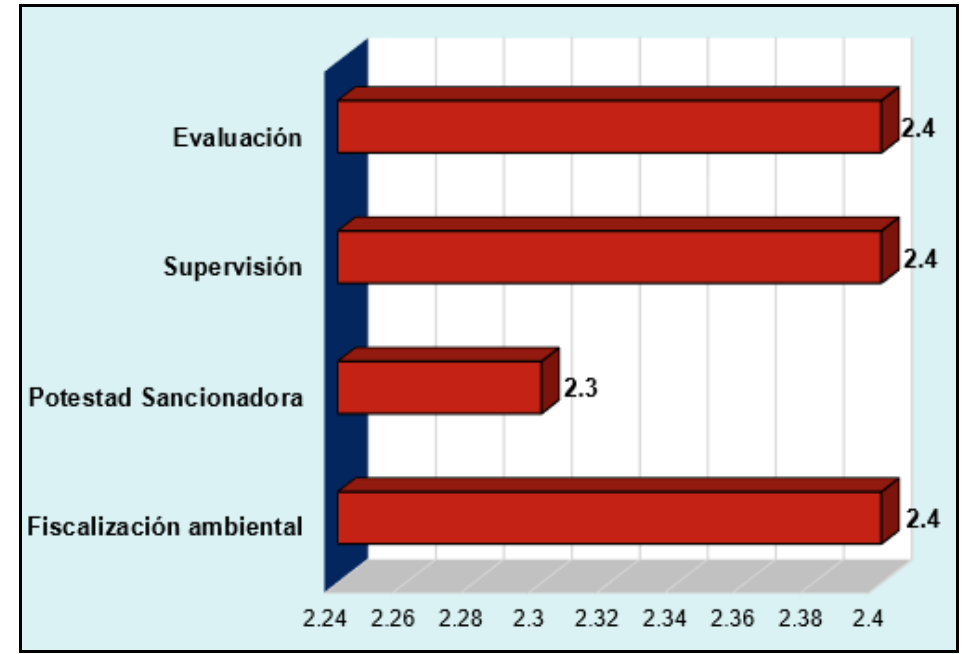

\subsection{Resultados y análisis de la gestión ambiental y su influencia en la fiscalización ambiental de la Municipalidad Provincial de San Martin}

Para el 5,3\% y el 36,8\% de los servidores de la Municipalidad Provincial de San Martín, la municipalidad nunca y casi nunca realiza una adecuada gestión ambiental. Asimismo, para el 5,3\% y el $50 \%$ de los servidores, la fiscalización ambiental se realiza de manera muy deficiente y deficiente respectivamente. Estos resultados mencionados se visualizan en la Tabla 5 y Figura 5 a continuación:

\section{Tabla 5}

Análisis de la gestión ambiental y su influencia en la fiscalización ambiental de la Municipalidad Provincial de San Martin

\begin{tabular}{llccccc}
\hline & & \multicolumn{4}{c}{ Fiscalización ambiental } & \\
\cline { 3 - 6 } & & Muy deficiente & Deficiente & Regular & Eficiente & Total \\
\cline { 3 - 6 } Gestión & Nunca & $2,6 \%$ & $2,6 \%$ & $0,0 \%$ & $0,0 \%$ & $5,3 \%$ \\
ambiental & Casi nunca & $2,6 \%$ & $26,3 \%$ & $7,9 \%$ & $0,0 \%$ & $36,8 \%$ \\
& A veces & $0,0 \%$ & $21,1 \%$ & $31,5 \%$ & $5,3 \%$ & $57,9 \%$ \\
\hline \multirow{2}{*}{ Total } & & $\mathbf{5 , 3 \%}$ & $\mathbf{5 0 , 0 \%}$ & $\mathbf{3 9 , 4 \%}$ & $\mathbf{5 , 3 \%}$ & $\mathbf{1 0 0 , 0 \%}$ \\
\hline
\end{tabular}




\section{Figura 5}

Análisis de la gestión ambiental y su influencia en la fiscalización ambiental de la Municipalidad Provincial de San Martin

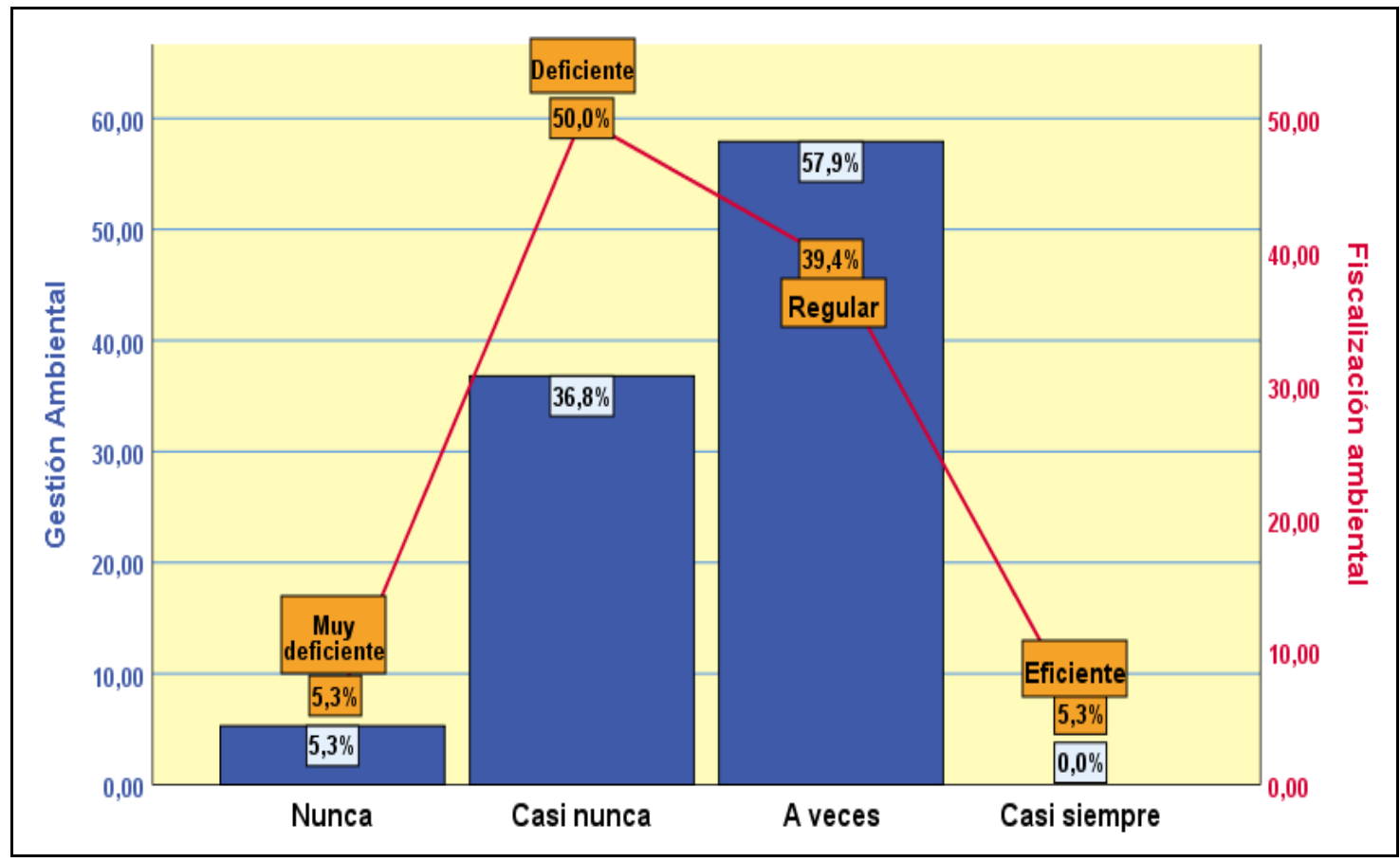

\subsection{1.- Resultados y análisis de la gestión ambiental y su influencia en la evaluación de la}

\section{Municipalidad Provincial de San Martin}

Para el 5,3\% y el 36,8\% de los servidores de la Municipalidad Provincial de San Martín, la municipalidad nunca y casi nunca realiza una adecuada gestión ambiental. También se encontró que, para el 7,9\% y el 47,4\% de los entrevistados, la evaluación ambiental se realiza de manera muy deficiente y deficiente respectivamente. Estos resultados mencionados se presentan en la Tabla 6 y Figura 6 a continuación:

\section{Tabla 6}

Gestión ambiental y su influencia en la evaluación de la Municipalidad Provincial de San Martin

\begin{tabular}{llccccc}
\hline & & \multicolumn{4}{c}{ Evaluación } & \multirow{2}{*}{ Total } \\
\cline { 3 - 6 } & \multirow{2}{*}{ Nunca } & Muy deficiente & Deficiente & Regular & Eficiente & \\
\cline { 3 - 6 } Gestión & $2,6 \%$ & $2,6 \%$ & $0,0 \%$ & $0,0 \%$ & $5,3 \%$ \\
ambiental & Casi nunca & $5,3 \%$ & $26,3 \%$ & $5,3 \%$ & $0,0 \%$ & $36,8 \%$ \\
& A veces & $0,0 \%$ & $18,4 \%$ & $34,2 \%$ & $5,3 \%$ & $57,9 \%$ \\
\hline \multirow{2}{*}{ Total } & $\mathbf{7 , 9 \%}$ & $\mathbf{4 7 , 4 \%}$ & $\mathbf{3 9 , 4 \%}$ & $\mathbf{5 , 3 \%}$ & $\mathbf{1 0 0 , 0 \%}$ \\
\hline
\end{tabular}

\section{Figura 6}

Gestión ambiental y su influencia en la evaluación de la Municipalidad Provincial de San Martin 


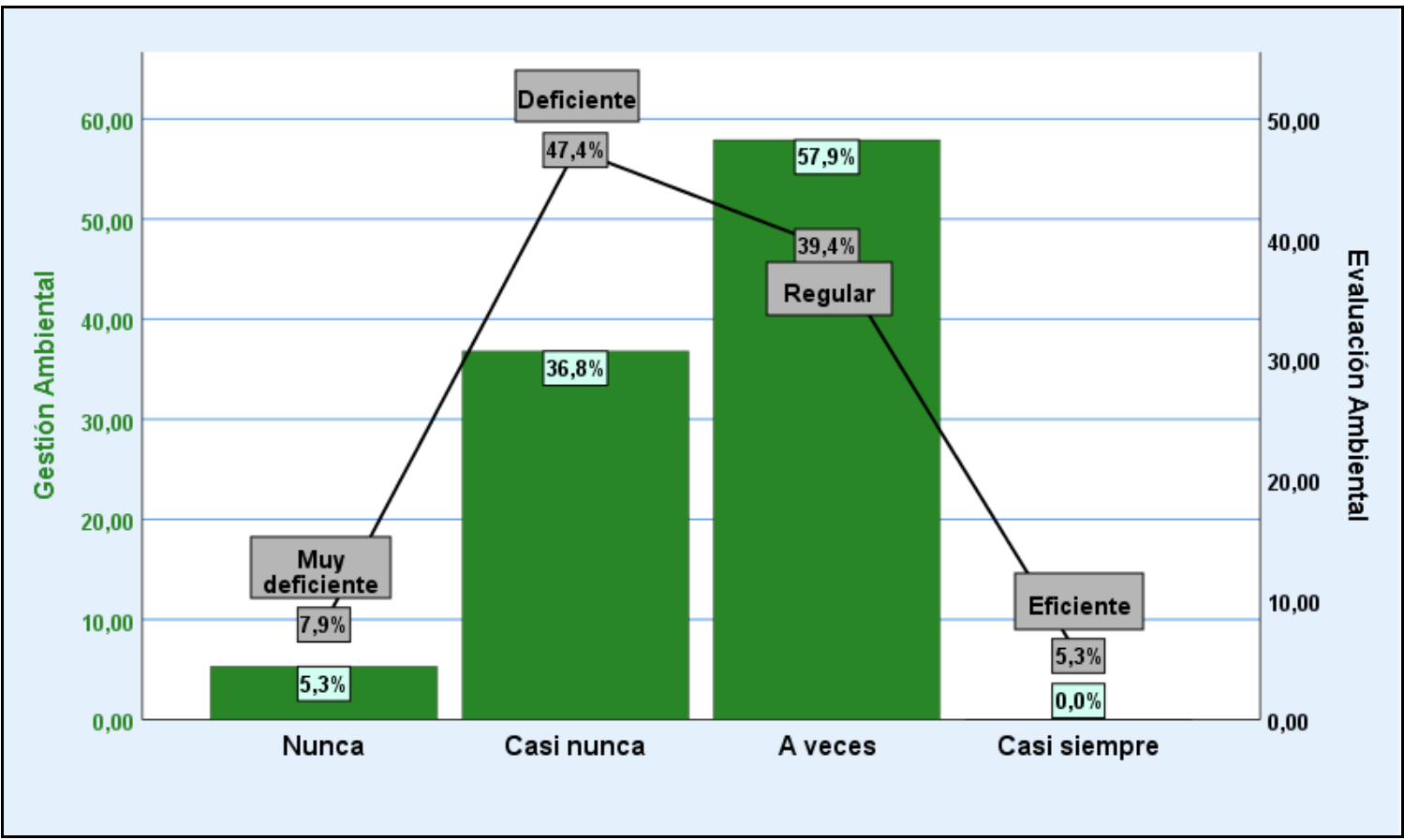

\subsection{2.- Resultados y análisis de la gestión ambiental y su influencia en la supervisión de la} Municipalidad Provincial de San Martin

Para el 5,3\% y el 36,8\% de los servidores de la Municipalidad Provincial de San Martín, la municipalidad nunca y casi nunca realiza una adecuada gestión ambiental. También se aprecia que, para el $7,9 \%$ y el $26,3 \%$ de, la supervisión ambiental se realiza de manera muy deficiente, y deficiente respectivamente. Estos resultados mencionados se presentan en la Tabla 7 y Figura 7 a continuación:

\section{Tabla 7}

Gestión ambiental y su influencia en la supervisión de la Municipalidad Provincial de San Martin

\begin{tabular}{llccccc}
\hline & & \multicolumn{4}{c}{ Supervisión } & \multirow{2}{*}{ Total } \\
\cline { 3 - 6 } & & Muy deficiente & Deficiente & Regular & Eficiente & \\
\cline { 3 - 6 } Gestión & Nunca & $2,6 \%$ & $2,6 \%$ & $0,0 \%$ & $0,0 \%$ & $5,3 \%$ \\
ambiental & Casi nunca & $5,3 \%$ & $15,8 \%$ & $15,8 \%$ & $0,0 \%$ & $36,8 \%$ \\
& A veces & $0,0 \%$ & $7,9 \%$ & $42,1 \%$ & $7,9 \%$ & $57,9 \%$ \\
\hline Total & & $\mathbf{7 , 9 \%}$ & $\mathbf{2 6 , 3 \%}$ & $\mathbf{5 7 , 9 \%}$ & $\mathbf{7 , 9 \%}$ & $\mathbf{1 0 0 , 0 \%}$ \\
\hline
\end{tabular}

\section{Figura 7}

Gestión ambiental y su influencia en la supervisión de la Municipalidad Provincial de San Martin 


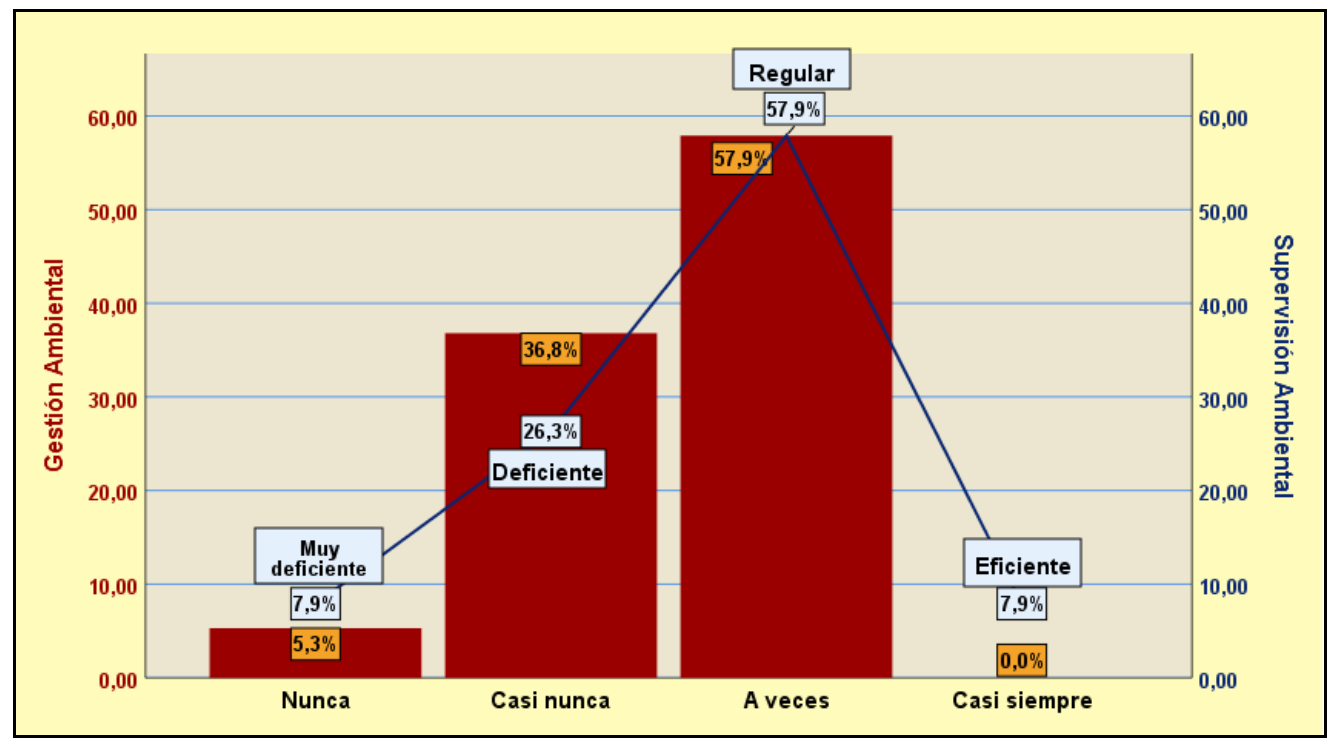

\subsection{3.- Resultados $y$ análisis de la gestión ambiental y su influencia en la potestad sancionadora de la Municipalidad Provincial de San Martin}

Para el 5,3\% y el 36,8\% de los servidores de la Municipalidad Provincial de San Martín, la municipalidad nunca y casi nunca realiza una adecuada gestión ambiental. Integrando a estos resultados, también se puede observar que, para el 15,8\% y el 55,3\% de los servidores de la Municipalidad Provincial de San Martin, la potestad sancionadora ambiental es muy deficiente, y deficiente respectivamente. Estos resultados mencionados se presentan en la Tabla 8 y Figura 8 a continuación:

\section{Tabla 8}

Gestión ambiental y su influencia en la potestad sancionadora de la Municipalidad Provincial de San Martin

\begin{tabular}{llccccc}
\hline & & \multicolumn{3}{c}{ Potestad Sancionadora } & \multirow{2}{*}{ Total } \\
\cline { 3 - 6 } & & Muy deficiente & Deficiente & Regular & Eficiente & \\
\cline { 3 - 6 } Gestión & Nunca & $5,3 \%$ & $0,0 \%$ & $0,0 \%$ & $0,0 \%$ & $5,3 \%$ \\
ambiental & Casi nunca & $7,9 \%$ & $26,3 \%$ & $2,6 \%$ & $0,0 \%$ & $36,8 \%$ \\
& A veces & $2,6 \%$ & $28,9 \%$ & $23,7 \%$ & $2,6 \%$ & $57,9 \%$ \\
\hline \multirow{2}{*}{ Total } & & $\mathbf{1 5 , 8 \%}$ & $\mathbf{5 5 , 3 \%}$ & $\mathbf{2 6 , 3 \%}$ & $\mathbf{2 , 6 \%}$ & $\mathbf{1 0 0 , 0 \%}$ \\
\hline
\end{tabular}

\section{Figura 8}

Gestión ambiental y su influencia en la potestad sancionadora de la Municipalidad Provincial de San Martin 


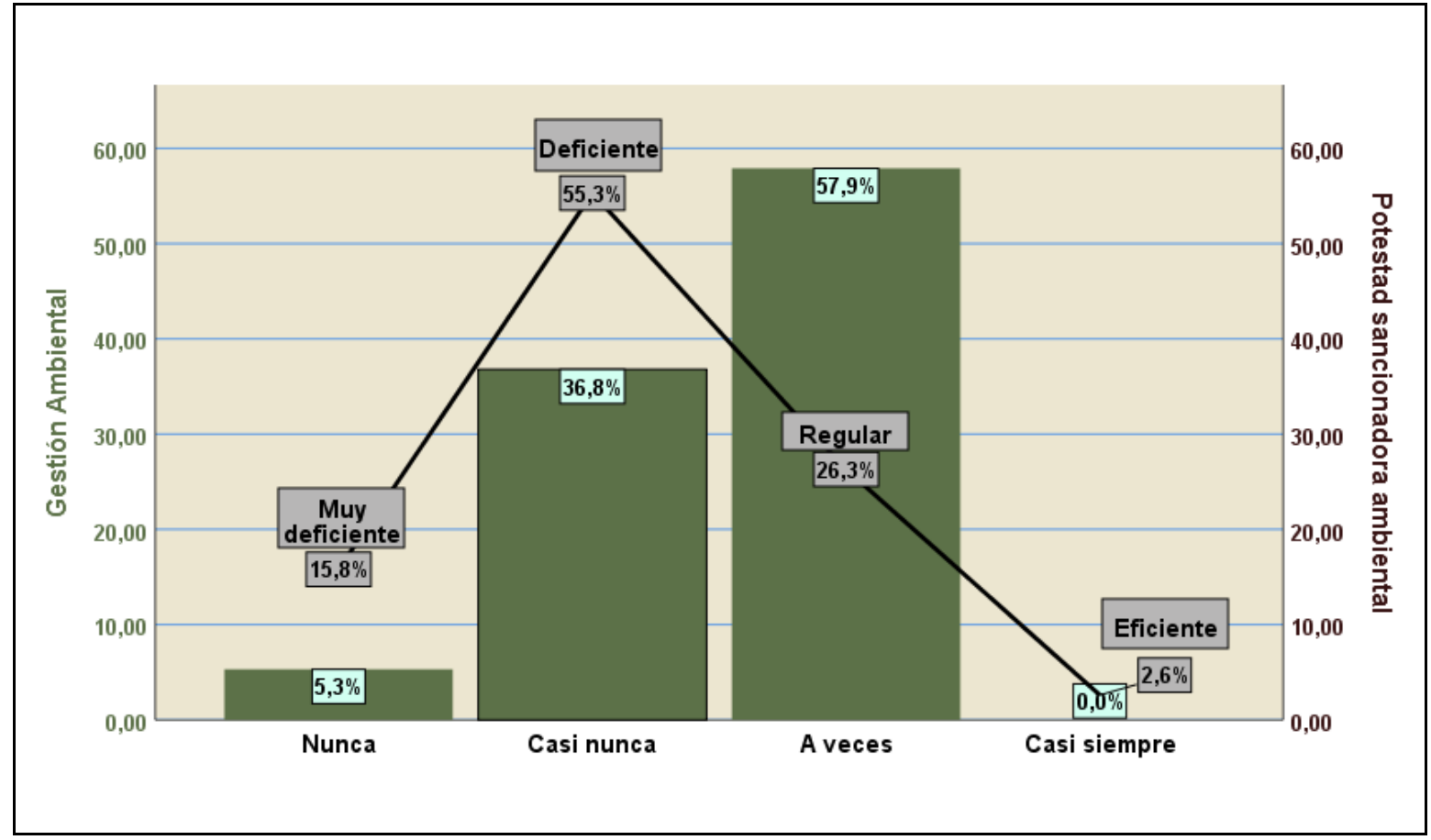

Finalmente, se presenta en la Tabla 9, la asociación entre la gestión ambiental y la fiscalización ambiental de la Municipalidad Provincial de San Martin 2021, donde se ha realizado la prueba chi cuadrado, encontrándose un $\mathrm{p}$ valor $=0,000<\alpha=0,05$, indicándonos que, si existe tal asociación. Por lo que se afirma que, la gestión ambiental se encuentra influyendo en la fiscalización ambiental.

\section{Tabla 9}

Análisis chi cuadrado de la gestión ambiental y la fiscalización ambiental de la Municipalidad Provincial de San Martin

\begin{tabular}{lccc}
\hline & Valor & df & $\begin{array}{c}\text { Significación asintótica } \\
\text { (bilateral) }\end{array}$ \\
\hline Chi-cuadrado de & $31,252^{\mathrm{a}}$ & 6 &, 000 \\
Pearson & 26,868 & 6 &, 000 \\
Razón de verosimilitud & 1 &, 000 \\
$\begin{array}{l}\text { Asociación lineal por } \\
\text { lineal }\end{array}$ & 20,751 & 1 & \\
N de casos válidos & 76 & & \\
\hline
\end{tabular}

a. 8 casillas $(66.7 \%)$ han esperado un recuento menor que 5. El recuento mínimo esperado es . 21 .

\subsection{Discusión}

En la investigación de (Ramírez, 2017) titulada "Importancia de la fiscalización ambiental en los factores que originan la aplicación de la sanción en materia ambiental firme en los procedimientos sancionadores de la Municipalidad Provincial de Tambopata Departamento de Madre de Dios", se encontró que: "En materia de supervisión 71\% fueron por manejo de residuos sólidos y 29\% acciones de supervisión por contaminación ambiental”. Se pueden apreciar grandes diferencias, puesto que, en la presente investigación, solo para el 10,5\% de los servidores encuestados, la supervisión en la protección del ambiente en la municipalidad se desarrolla de 
manera entre eficiente, y muy eficiente; es decir, existe diferencias con lo encontrado por (Ramírez, 2017), donde se aprecia claramente que se realiza la supervisión en mayor índice porcentual.

En la investigación de (Rodríguez, 2020) denominada "Gestión ambiental y ecoeficiencia en la Municipalidad Provincial de Moyobamba, 2020", se encontró lo siguiente: "El 61,6\% consideró que la gestión ambiental en la municipalidad es regular, el $20,5 \%$ que es malo y el $17,8 \%$ buena". En el presente estudio, según el 42,1\% de los servidores de la Municipalidad Provincial de San Martín, la municipalidad no realiza una adecuada gestión ambiental, es decir, en la Municipalidad Provincial de San Martin existe una peor gestión ambiental, que, en la Municipalidad Provincial de Moyobamba, según lo encontrado por (Rodríguez, 2020).

También, en la investigación de (Rodríguez, 2020) denominada "Gestión ambiental y ecoeficiencia en la Municipalidad Provincial de Moyobamba, 2020", se halló lo siguiente: "En cuanto al servicio social el 46,6 opinó que es regular y el 31,5\% que es malo". Encontrándose que, en la presente investigación para el 57,9\% de los entrevistados, la municipalidad no desarrolla actividades productivas de inclusión social aprovechando los recursos naturales, encontrándose diferencias con lo hallado por (Rodríguez, 2020), donde el servicio social ambiental se desarrolla de mejor manera.

En la tesis de (Roca, 2019) titulada "Auditoría ambiental y su efecto en la evaluación de la gestión ambiental de la Municipalidad Provincial de Huamanga, 2018”, se encontró lo siguiente: "El 73\% afirman que la toma de decisiones si se tiene en cuenta la repercusión de sus actividades ambientales en la sociedad y el $27 \%$ respondieron que no se tiene en cuenta la repercusión de sus actividades ambientales en la sociedad". En la Municipalidad Provincial de San Martin, sólo el 2,6\% de los servidores encuestados, indicaron que la municipalidad siempre genera la información ambiental para la toma de decisiones, porcentaje menor a lo encontrado por (Roca, 2019), en la Municipalidad Provincial de Huamanga.

También, en la investigación de (Roca, 2019) titulada "Auditoría ambiental y su efecto en la evaluación de la gestión ambiental de la Municipalidad Provincial de Huamanga, 2018", se halló lo siguiente: "El 41\% afirman que siempre reciben capacitación en materia ambiental; el 39\% respondieron que ocasionalmente; el $10 \%$ respondieron que a veces, y el $10 \%$ respondieron que nunca reciben capacitación en materia ambiental". En la Municipalidad Provincial de San Martin, se encontró que, solo el 2,6\% de los entrevistados, indicaron que, la municipalidad casi siempre capacita al personal para la gestión ambiental, presentándose diferencias con lo encontrado por (Roca, 2019), donde existe mayor porcentaje que indicó si reciben capacitación en materia ambiental en la Municipalidad Provincial de Huamanga.

En la investigación de (Ushiñahua, 2019) sobre la "Gestión ambiental y su relación con el manejo integral de residuos sólidos en la Municipalidad Provincial de San Martín, 2018", se encontró lo siguiente: "El $64 \%$ de los resultados calificó a la dimensión fiscalización como Inadecuada". En el presente estudio, para el 94,7\% de los servidores de la Municipalidad Provincial de San Martín, la fiscalización ambiental no se realiza de manera eficiente, encontrándose diferencias con lo hallado por (Ushiñahua, 2019).

\section{CONCLUSIÓN}

PRIMERA: La gestión ambiental se encuentra asociada a la fiscalización ambiental de la Municipalidad Provincial de San Martin. Esta conclusión se refleja en el 97,4\% de los servidores, quienes indicaron que la municipalidad, no siempre genera la información ambiental para la toma de decisiones. También, el 89,5\% indicó que, la municipalidad, no siempre toma acciones para fortalecer la educación ambiental. Por su parte, el 97,4\% de los servidores, indicaron que la municipalidad no siempre promociona adecuadamente la inversión sostenible. 
SEGUNDA: La gestión ambiental se encuentra asociada con la evaluación de la Municipalidad Provincial de San Martin. Encontrándose que, según el 97,4\% de los entrevistados, la identificación de los factores que influyen en la calidad del ambiente no es eficiente. Por su parte, el 94,7\% señaló que, la evaluación ambiental no se realiza de manera eficiente.

TERCERA: La gestión ambiental está asociada con la supervisión de la Municipalidad Provincial de San Martin. Asimismo, se tiene que para el 100\% de los entrevistados, el cumplimiento de las obligaciones ambientales en el proceso de fiscalización no es eficiente.

CUARTA: La gestión ambiental se encuentra asociada con la potestad sancionadora de la Municipalidad Provincial de San Martin. Encontrándose que, para el 97,4\% de los entrevistados, el estudio de las posibles infracciones administrativas a nivel ambiental, no es eficiente. También, el 97,4\% señaló que la implementación de las medidas correctivas según la fiscalización ambiental no es eficiente.

\section{CONSIDERACIONES FINALES}

Siendo la temática de gestión ambiental y fiscalización ambiental de gran importancia en la actualidad, no se han presentado limitaciones teóricas en este estudio, ya que se pudo obtener información de los diversos repositorios universitarios (pre y posgrado), así como información de articulo indexados en revistas científicas, tanto de carácter nacional como internacional. Respecto a las bases teóricas en el Perú se continúa fortaleciendo el tema, esto a través de diversos autores, y la postura de actuación institucional de la entidad rectora, como el Organismo de Evaluación y Fiscalización Ambiental. Tampoco se han presentado limitaciones metodológicas en este estudio, siendo que la misma cumple con los criterios del rigor científico tales como el valor de verdad, su aplicabilidad, su consistencia y su neutralidad; además de poder ser replicable la metodología en otras entidades, a fin de obtener información sobre las variables. Finalmente, a partir de los resultados de la investigación, pueden surgir investigaciones respecto al control normativo y/o técnico o nivel de cumplimiento de la gestión ambiental y fiscalización ambiental en entidades públicas, para garantizar el derecho a un ambiente sano y equilibrado conforme señala la Constitución Política del Perú.

\section{REFERENCIAS}

Araujo, L., \& Casanova, M. (2018). "El grado de cumplimiento de la fiscalización ambiental en las municipalidades distritales de la provincia de Trujillo durante los años 2013 al 2017". Universidad Nacional de Trujillo, Trujillo,Perú. Obtenido de http://dspace.unitru.edu.pe/bitstream/handle/UNITRU/10517/AraujoBacon_L\%20\%20CasanovaMejia_M.pdf?sequence=1\&isAllowed=yArd

Armas, G. (2020). La gestión ambental y sus efectos en el desarrollo sostenible. Revista Centroamericana de Administración Pública, vol.78, pp.42-66. doi:https://doi.org/10.35485/rcap78_2

Comisión Multisectorial. (2012). Ejes estratégicos de la Gestión Ambiental. Lima. Obtenido de https://cdn.www.gob.pe/uploads/document/file/307801/EJESESTRATEGICOS-DE-LA-GESTIONAMBIENTAL.pdf

George, D., \& Mallery, P. (2020). IBM SPSS Statistics 26 Step by Step. New York: Routledge.

Gómez, H. (2017). La fiscalización ambiental del OEFA: características de un modelo de ejercicio de la potestad sancionadora que armoniza con la inversión. Obtenido de https://repositorioacademico.upc.edu.pe/bitstream/handle/10757/621638/Tesis+Grado+de+Magi\%20ster,+Hug o+Go\%20mez,+para+biblioteca+de+UPC+(2).pdf;jsessionid=92EC877DA9BCAAC312B0D42B05A30B5B?s equence $=1$

González, M. (2018). Evaluación integral del proceso de fiscalización ambiental realizado por el Organismo de Evaluación Ambiental en el Perú. Obtenido de https://repositorio.lamolina.edu.pe/bitstream/handle/20.500.12996/3216/gonzalez-roca-micaelacayetana.pdf?sequence $=1 \&$ isAllowed $=y$ 
Gutiérrez, C. (2020). La Gestión Ambiental en tiempos de pandemia. Chile: Konrad Adenauer Stiftung. Obtenido de https://www.kas.de/documents/273477/8706787/Descarga+el+art\%C3\%ADculo+aqu\%C3\%AD++La+Gesti\%C3\%B3n+Ambiental+en+Tiempos+de+Pandemia.pdf/b6dc26b2-63a7-4c2c-f4d897f0ad41e8a?version $=1.1 \& \mathrm{t}=1586370695215$

Instituto Nacional de Estadística e Informática. (2020). Perú: Anuario de Estadísticas Ambientales 2020. Perú: INEI. Obtenido de https://www.inei.gob.pe/media/MenuRecursivo/publicaciones_digitales/Est/Lib1760/libro.pdf

Ley General del Ambiente. (2017). LEY No 28611. Lima,Perú. Obtenido de https://www.minam.gob.pe/wpcontent/uploads/2017/04/Ley-N\%C2\%B0-28611.pdf

Ministerio del Ambiente. (2012). Glosario de términos para la gestión ambiental Peruana. Lima. Obtenido de http://siar.minam.gob.pe/puno/sites/default/files/archivos/public/docs/504.pdf

Ministerio del Ambiente. (2016). La fiscalización ambiental en el Perú. Lima. Obtenido de https://sinia.minam.gob.pe/documentos/fiscalizacionambiental-peru-2011-2015-fortaleciendo-cimientos

Municipalidad Provincial de San Martín. (2020). Cuadro para asignación de personal provisional (CAP-P). Oficina de Presupuesto y Racionalización, Tarapoto, San Martín. Obtenido de https://www.mpsm.gob.pe/public/uploads/documentos/2020-cap-2020.pdf

Organismo de Evaluación y Fiscalización Ambiental. (2012). Ley del Sistema Nacional de Evaluación y Fiscalización Ambiental. Ley $\mathrm{N}^{\circ} 29325$. Obtenido de https://www.oefa.gob.pe/?wpfb_dl=12165

Organismo de Evaluación y Fiscalización Ambiental. (2018). Manual de competencias de las entidades de Fiscalización Ambiental. Lima,Perú. Obtenido de https://www.oefa.gob.pe/?wpfb_dl=33950

Oyague, E., Yaja, A., \& Franco, P. (2020). Efectos ambientales del confinamiento debido a la pandemia de COVID-19: evaluación conceptual y análisis de datos empíricos en Tacna, marzo-abril 2020. Ciencia \& Desarrollo, Vol.19(26), pp18. doi:doi.org/10.33326/26176033.2020.26.901

Programa de Política y Gestión Ambiental - SPDA. (2016). La Participación ciudadana en los procesos de evaluación de impacto amniental. Lima: Sociedad Peruana de Derecho Ambiental. Obtenido de http://smia.munlima.gob.pe/uploads/documento/2c786d5fc0308fed.pdf

Programa Puentes UC. (2019). Gestión Ambiental Local en Municipios. Programa Puentes UC, Chile. Obtenido de https://static1.squarespace.com/static/5d72e113526e831468643361/t/5e972e75c7803229caed0f14/1586966203 197/GESTIO\%CC\%81N+AMBIENTAL+LOCAL+EN+MUNICIPIOS.pdf

Ramírez, E. (2017). "Importancia de la fiscalización ambiental en los factores que originan la aplicación de la sanción en materia ambiental firme en los procedimientos sancionadores de la municipalidad provincial de Tambopata departamento de Madre de Dios". Universidad Andina del Cusco, Madre de Dios. Obtenido de http://repositorio.uandina.edu.pe/bitstream/UAC/1004/3/Eberth_Tesis_bachiller_2017.pdf

Resolución Ministerial N²47-2013-MINAM. (2013). Lima. Obtenido de https://www.minam.gob.pe/wpcontent/uploads/2014/02/RM-N\%C2\%BA247-2013-MINAM.pdf

Roca, M. (2019). Auditoría ambiental y su efecto en la evaluación de la gestión ambiental de la Municipalidad Provincial de Huamanga, 2018. Universidad Católica los Ángeles Chimbote, Ayacucho, Perú. Obtenido de http://repositorio.uladech.edu.pe/bitstream/handle/123456789/11353/AUDITORIA_AMBIENTAL_EVALUA CION_EXAMEN_GESTION_AMBIENTAL_ROCA_GOMEZ_\%20MARIA_DEL_PILAR.pdf?sequence=1 \&isAllowed $=\mathrm{y}$

Rodríguez, B. (2020). "Gestión ambiental y ecoeficiencia en la Municipalidad Provincial de Moyobamba, 2020". Universidad César Vallejo, Tarapoto, Perú. Obtenido de https://repositorio.ucv.edu.pe/bitstream/handle/20.500.12692/50517/Rodr\%c3\%adguez_RBESD.pdf? sequence $=1 \&$ is Allowed $=\mathrm{y}$

Roggeroni, V. (2014). Análisis de la Aplicación del Sistema Local de Gestión Ambiental de la Provincia de Mariscal Ramón Castilla partir de la Norma ISO 14001. Universidad de Manizales, Colombia. Obtenido de https://ridum.umanizales.edu.co/xmlui/bitstream/handle/20.500.12746/1752/TESIS\%20VANESSA\%20ROGG ERONI.pdf?sequence $=1$ \&isAllowed $=\mathrm{y}$

Rosado, E. (2021). "Influencia de la fiscalización ambiental en la gestión sostenible de los recursos naturales del Gobierno Regional de Ucayali 2018". Universidad Nacional de Ucayali, Pucallpa. Obtenido de http://repositorio.unu.edu.pe/bitstream/handle/UNU/4942/B69_UNU_MAESTRIA_2021_TM_ERNESTOROSADO.pdf?sequence=1\&isAllowed=y

Schoemaker. (2017). Glosario Ambiental:Conociendo los términos ambientales usados en zonas mineras. Lima: Caja de Herramientas Ambientales. Obtenido de https://democraciaglobal.org/wp-content/uploads/tomo1_optimize.pdf

Ushiñahua, M. (2019). "Gestión ambiental y su relación con el manejo integral de residuos sólidos en la Municipalidad Provincial de San Martín, 2018”. Universidad César Vallejo, Tarapoto. Obtenido de https://hdl.handle.net/20.500.12692/31290 